provincial reference laboratory (Laboratoire de santé publique du Québec) and the NG-MAST typing is performed at the National Microbiology Laboratory. Cases are classified as retained (presence of all definition criteria) or suspected (not meeting all criteria but reinfection unlikely). Cases classifications are validated by a group of experts. Lack of re-exposure is based on the respondent's reported sexual history between the first treatment and the test of cure (TOC). Case definitions are consistent with those of Quebec and Canadian sentinel surveillance network for gonococcal infection.

Results Between November 2014 and December 2018, 44 cases of possible treatment failures were reported. After exclusion of 9 cases, 35 were analysed (25 classified as retained and 10 as suspected). There were 10 women, 24 men $(68 \%$ MSM) and one trans person. Pharynx was identified as site of treatment failure for 14 cases (40\%). AST were available for 23 cases (66\%): 78\% were resistant to ciprofloxacin and $43 \%$ to azithromycin. All strains were susceptible to cephalosporins, but one strain showed reduced susceptibility to cefixime. Eleven cases (31\%) received azithromycin monotherapy as initial therapy.

Conclusion Treatment failure exist and is not always related to documented resistance. This analysis probably underestimates the real extent of treatment failures since it requires TOC that are not systematically collected. Reinfection cannot be completely excluded.

Disclosure No significant relationships.

\section{P639 MOLECULAR MARKERS TO PREDICT CEFIXIME DECREASED SUSCEPTIBILITY OF NEISSERIA GONORRHOEAE: A GLOBAL REVIEW}

${ }^{1}$ Xiaomeng Deng*, ${ }^{2}$ Lao-Tzu Allan-Blitz, ${ }^{3}$ Jeffrey Klausner. 'David Geffen School of Medicine, Los Angeles, USA; ${ }^{2}$ Brigham and Women's Hospital, Department of Medicine, Boston, USA; ${ }^{3}$ UCLA - David Geffen School of Medicine, Infectious Diseases, Los Angeles, USA

10.1136/sextrans-2019-sti.707

Background In the last two decades, there have been numerous reports worldwide of Neisseria gonorrhoeae (NG) infections with clinical treatment failure to cefixime. Mutation in multiple NG genes including pen $\mathrm{A}, m t r \mathrm{R}$, pil $\mathrm{Q}$, pen $\mathrm{B}$, and pon $\mathrm{A}$, have been associated with cefixime decreased susceptibility and resistance, however, no single mutation has been identified as necessary or sufficient.

Methods We performed a systematic review of all PubMedpublished articles from $01 / 01 / 1995$ to $11 / 01 / 2018$ that reported molecular characteristics of decreased susceptibility of NG to cefixime. We summarized the findings and made suggestions for the development of a molecular-based NG assay to predict cefixime susceptibility. Based on clinical outcome data, we defined a minimum inhibitory concentration (MIC) $\geq 0.12 \mathrm{ug} / \mathrm{mL}$ as the cutoff for decreased susceptibility to cefixime. For a wild-type (non-mutated) sequence comparison, we used the pen $\mathrm{A}$ peptide sequence of NG reference strain M32091.

Results We found 74 articles, of which we excluded 49 due to incomplete information. Among the 25 articles included, there were 415 reported NG strains with reduced susceptibility to cefixime from 22 countries. Two types of penA alterations accounted for $99.5 \%$ (413/415) of strains with decreased susceptibility to cefixime: (1) mosaic penA, which can be identified by mutations at amino acid position $375-377$ or (2) non-mosaic penA but with at least one critical amino acid substation at position 501, 542, or 551. The other two strains with $\mathrm{MIC} \geq 0.125 \mu \mathrm{g} / \mathrm{mL}$ were found in Spain in 2013 with a non-mosaic pen $\mathrm{A}$ sequence but no alteration at amino acid position 501,542 or 551 .

Conclusion We conducted a systematic review of published reports of over $400 \mathrm{NG}$ strains with decreased susceptibility to cefixime. We identified a combination of sequences in the mosaic and non-mosaic regions of the pen $\mathrm{A}$ gene that if wildtype (non-mutated) may serve as reliable and sensitive markers to predict cefixime susceptibility globally.

Disclosure No significant relationships.

\section{P640 TARGETING MURI PROTEIN TO COMBAT DRUG RESISTANCE IN NEISSERIA GONORRHOEAE USING HOMOLOGY MODELING AND DRUG DOCKING STUDIES}

Ravi Kant, Prakash Jha, Alka Pawar, Madhu Chopra, Daman Saluja. University of Delhi, New Delhi, Dr. B R Ambedkar Center for Biomedical Research (ACBR), New Delhi, India

\subsection{6/sextrans-2019-sti.708}

Background Neisseria gonorrhoeae, a causative agent of gonorrhea, has developed resistance to most of the drugs and hence aptly declared as 'Superbug'. Glutamate racemase (MurI) considered as an important drug target. Therefore, identification of novel drugs for the treatment of gonorrhea is urgently required.

Methods The amino acid sequence of MurI of Neisseria gonorrhoeae (YP_208550) was retrieved from NCBI. Based on query coverage, e-score and percentage similarity, 3OUT (glutamate racemase from Francisella tularensis) was selected as template after PDB BLAST, homology model was generated by Modeller programme of Discovery Studio 4.0.Best model was selected based on DOPE score and PDF energy score and further verified by Verify-3D protocol and Ramachandran Plot. Receptor binding site was identified after superimposition of template structure and modelled structure and the co-crystalized ligand of the template was docked into the modeled MurI structure. Based on docking score, best pose was selected and receptor-ligand pharmacophore model was generated.

Results The best homology model generated was selected based on the verify score of 107.93 from Verify 3D program of Discovery Studio 4.0. Validation of the selected model by Ramachandran plot showed214 residues (91.8\%) fall in most favored region. RMSD of 0.2475 A0was generated by superimposition of query and template structures. Quality factorof $84 \%$ for the protein models was obtained using ERRAT.Six pharmacophores were generated using best docking pose between D-glutamate and MurI. These were subjected to virtual screening with DrugBank database.586 hits so obtained were filtered by fit value of 3.51 which resulted in 268 hits. These 268 hits were further subjected to Lipinski and veber filter followed by ADMET, gave 73 hits. These were subjected to energy minimization and docking to obtain the best hits.

Conclusion The study identifies potential compounds that interact with active site of MurI protein, opening new avenues for the treatment option against multidrug resistant strains. Disclosure No significant relationships. 